\title{
Prevalence and Stability of Qualitative Qol Resistance in Populations of Venturia inaequalis in the Northeastern United States
}

Zachary A. Frederick and Sara M. Villani, Department of Plant Pathology and Plant-Microbe Biology, Cornell University, Geneva, NY 14456; Daniel R. Cooley, Department Plant, Soil, and Insect Science, University of Massachusetts, Amherst 01003; Alan R. Biggs, Kearneysville Tree Fruit Research and Education Center, West Virginia University, Kearneysville 25443; and Jessica J. Raes and Kerik D. Cox, Department of Plant Pathology and Plant-Microbe Biology, Cornell University

\begin{abstract}
Frederick, Z. A., Villani, S. M., Cooley, D. R., Biggs, A. R., Raes, J. J., and Cox, K. D. 2014. Prevalence and stability of qualitative QoI resistance in populations of Venturia inaequalis in the northeastern United States. Plant Dis. 98:1122-1130.

Quinone-outside-inhibitor (QoI) fungicides are a safe and effective means of managing apple scab caused by Venturia inaequalis. To determine the prevalence of both quantitative (partial) and qualitative (complete) QoI resistance in $V$. inaequalis in the northeastern United States, we sampled single-lesion conidial isolates $(n=4,481)$ from 120 commercial and research orchards from 2004 to 2011 with a range of exposure to QoI fungicides from none to several applications a year. In all, $67 \%$ of these orchard populations of $V$. inaequalis were sensitive to QoI fungicides, 28\% exhibited QoI practical resistance, and 5\% were not sensitive QoI fungicides but had not become practically resistant. Isolates with qualitative QoI resistance, conferred by the G143A cytochrome $b$ gene mutation, were found in 13 of the 34 QoI-resistant or-

chard populations. To evaluate the stability of the G143A mutation, 27 isolates were selected from different orchard populations to represent the scope of regional populations. These isolates were subcultured continuously in the presence or absence of the QoI fungicide trifloxystrobin. All isolates that initially possessed qualitative resistance maintained the resistant genotype (G143A) for six transfers over 6 months in both the absence and presence of trifloxystrobin. Given the observed QoI resistance in orchard populations of $V$. inaequalis and the stability of the G143A mutation in individual isolates, apple scab management paradigms must encompass strategies to limit selection of QoI resistance in the sensitive orchard populations remaining in the region.
\end{abstract}

Apple scab, caused by the ascomycete fungal pathogen Venturia inaequalis, is one of the most economically important diseases of apple $(2,14,23)$. The disease is most severe in temperate climates where weather conditions during spring are conducive to infection $(12,23)$. Infections occur when temperatures are over $1^{\circ} \mathrm{C}$ and leaves are wet for at least $6 \mathrm{~h} \mathrm{(24).} \mathrm{Marketable} \mathrm{fruit} \mathrm{losses} \mathrm{can}$ generally range from 50 to $100 \%$ of the total fresh market harvest if proper chemical management programs are not used in commercial orchards in temperate zones during a season with typical weather conditions $(2,12,14)$.

Despite the existence of host resistance in a few cultivars, successful management of apple scab is primarily achieved through the use of protectant and systemic fungicides $(1,23)$. Of these fungicides, quinone-outside-inhibitors (QoIs), have proven to be safe and highly effective for apple scab management $(9,26)$. The antifungal activity of QoIs was first noted in the 1970s (3), and they were first used in apple for apple scab management in the mid- to late 1990s $(18,27)$.

QoI fungicides prevent fungal respiration by inhibiting electron transfer at the ubiquinol-oxidation (Qo) site of the mitochondrial cytochrome bc1 enzyme III complex $(5,9,22)$. One of the most common mechanisms of QoI resistance described for fungal pathogens is a single point mutation that codes for alanine instead of glycine at position 143 (G143A) in the cytochrome $b$ (cyt b) gene (5), which imparts a vertical, qualitative, or complete resistance response whereby isolates are highly resistant to QoI fungicides (23). Previously, qualitative QoI resistance was reported in several orchard populations of $V$. inaequalis in Michigan (20), and was previously reported in a single $V$. inaequalis population in New

Corresponding author: K. D. Cox, E-mail: kdc33@cornell.edu

Accepted for publication 21 February 2014.

http://dx.doi.org/10.1094/PDIS-10-13-1042-RE

(c) 2014 The American Phytopathological Society
York (7). Other point mutations, both naturally and artificially induced, also confer qualitative resistance to QoI fungicides in other fungal pathogens $(15,16,22)$; however, none of these has been reported in $V$. inaequalis to date. Additionally, there is an alternative respiration or cyanide insensitive pathway (9), which may influence the response of fungi to QoI fungicides $(8,33)$. This pathway allows fungi to bypass the inhibited respiration pathway and persist through exposure to QoIs, as shown for Magnaporthe grisea (25), Septoria triciti (34), and Botrytis cinerea (30). This alternative respiration pathway becomes operative in $V$. inaequalis following exposure to high doses of QoI fungicides (27). Given the importance of qualitative resistance phenomena in several pathosystems, information on the stability of the G143A mutation in the absence of selective pressure is of key importance for understanding the persistence of qualitative resistance in $V$. inaequalis. Although investigations into the stability of such $c y t b$ gene mutations have been carried out for a number of plant-pathogenic fungi $(25,30,34)$, the stability of the G143A mutation in $V$. inaequalis is largely unknown. Information on the stability of this mutation within isolates of $V$. inaequalis would be the first step in understanding the persistence of qualitative resistance in an orchard population.

In addition to qualitative resistance, there appears to be a quantitative (polygenic or partial) resistance response to QoI fungicides in $V$. inaequalis whereby a collection of isolates may display a range of insensitivity phenotypes below the point at which the alternative respiration pathway becomes activated (18). Such responses would include those resulting from unknown factors imparting a minor level of fungicide insensitivity outside of the context of $c y t b$ gene mutation. However, little is known about the molecular basis of quantitative resistance to QoI fungicides in $V$. inaequalis or other pathosystems. It was been hypothesized in this seminal report that this quantitative resistance precedes QoI qualitative resistance in $V$. inaequalis in commercial orchard populations (18). In such a scenario, isolates with quantitative resistance could emerge in a population prior to the onset of QoI practical resistance (18), which is defined as greatly diminished levels of 
disease control (i.e., a control failure) following proper QoI fungicide application under field conditions (17).

To date, the prevalence and stability of qualitative resistance in populations of $V$. inaequalis in the northeastern United States is not well understood. Moreover, as practical resistance to QoI fungicides is reported to authors, the contribution of isolates with quantitative resistance or qualitative resistance to the development of practical resistance is warranted. Hence, the objectives of this study were to (i) determine the prevalence of quantitative and qualitative QoI resistance in orchard populations of $V$. inaequalis in the northeastern United States and (ii) assess the stability of qualitative QoI resistance in V. inaequalis in the absence of QoIinduced selection pressure.

\section{Materials and Methods}

In vitro sensitivity of orchard populations of $V$. inaequalis to the QoI fungicide trifloxystrobin. From 2004 to 2011, isolates of $V$. inaequalis were collected from 120 apple orchard populations located in the northeastern United States, including populations from 94 commercial, 16 research, and 10 baseline orchards in multiple states (Table 1). Baseline orchards are defined as orchard plantings never having exposure to site-specific fungicide chemistries, including the anilinopyrimidines, sterol biosynthesis or demethylation inhibitors, QoIs, benzimidazoles, dodine, and succinate dehydrogenase inhibitors. Of the 120 total orchard populations, 24 were sampled in multiple years (Table 1).

To obtain isolates for each of the 120 orchards, 50 to 100 symptomatic leaves were arbitrarily collected from trees throughout the planting. From each collection of leaves, 50 distinct individual leaf lesions arising from single-spore infections, composed of clonal conidia $(18,24)$, were identified and processed for the evaluation of quantitative resistance (18) to the QoI fungicide trifloxystrobin. Each sporulating leaf lesion was excised with a cork borer $(7 \mathrm{~mm}$ in diameter), placed in $1.2 \mathrm{ml}$ of distilled water, and shaken for 60 $\mathrm{s}$ to rinse conidia from the lesion. The clonal conidia in the resulting suspension were considered a single isolate, which was termed a single-lesion conidial isolate, as described by Köller et al. $(18,19)$.

The individual isolates were evaluated for QoI resistance phenotypes using a microscopy-aided mycelial relative growth assay for V. inaequalis previously published by Köller et al. $(18,19)$. To summarize, this procedure involved applying 100- $\mu$ l aliquots of each single-lesion conidial suspension $\left(10^{4}\right.$ conidia/ml $)$ onto the surface of potato dextrose agar (PDA; Difco Laboratories) amended with a discriminatory dose of trifloxystrobin $(0.02 \mu \mathrm{g} / \mathrm{ml}$ analytical standard; Sigma-Aldrich; 18) and to PDA without trifloxystrobin. This discriminatory dose was chosen based on previously published evaluations of the quantitative (polygenic or partial) resistance response to QoI fungicides in V. inaequalis (18). Quantitative (polygenic or partial) resistance response was described (18) as insensitivity phenotypes observed when isolates were exposed to doses of QoIs below the point at which the alternative respiration pathway becomes activated. These resistance responses would encompass the contributions of both unknown minor gene factors and the $c y t b$ gene mutations, if present. Streptomycin sulfate (50 $\mu \mathrm{g} / \mathrm{ml}$, Sigma-Aldrich,) and chloramphenicol (50 $\mu \mathrm{g} / \mathrm{ml}$, SigmaAldrich) were added to all media to suppress bacteria and yeast fungi. Cultures were incubated for 7 days at 23 to $25^{\circ} \mathrm{C}$. Mean percent relative growth $(\% \mathrm{RG})$ for each isolate was determined by measuring the mean growth of five colonies, each originating from a single conidium, in the presence of trifloxystrobin relative to the mean growth of five such colonies on the PDA medium without trifloxystrobin. All colonies were measured at the point of their largest expansion using a SPOT Idea digital camera with the SPOT Imaging Basic software package (Diagnostic Instruments Inc.) attached to an Olympus SZX12 stereo scope ( $\times 70$ to 120 ; Olympus America Inc.).

Quantitative QoI sensitivity responses in the form of mean \% RG values were generated for each isolate in each of the 120 orchard populations. Each orchard population was subsequently classified as having or lacking practical resistance to trifloxystrobin by comparing the distribution of sensitivity responses (mean \% RG values of isolates) for the orchard population in question with standard orchard populations with known practical resistance or sensitivity to QoI fungicides. This method for comparing and classifying the practical resistance status for populations of $V$. inaequalis on the basis of quantitative fungicide resistance response was previously described by Köller et al. (19). Practical resistance or sensitivity was determined directly by examining fungicide application practices for each standard orchard and assessing apple scab symptom development following QoI use. Orchard population 33 had disease incidence of $0.0 \%$ apple scab on fruit and leaves at harvest following four applications of Flint WG (a.i. trifloxystrobin) in 2005 and was used as the sensitive standard population (mean isolate growth rate $0.71 \mathrm{~mm} /$ day). Orchard population $118 \mathrm{had}$ disease incidence of $40.6 \%$ apple scab on fruit at harvest following four applications of Flint WG (a.i. trifloxystrobin) and was used as the resistant standard (mean isolate growth rate $0.74 \mathrm{~mm} /$ day). Comparisons of isolate sensitivity distributions for each of the 120 orchard populations to the practical resistant and sensitive standards were made using the nonparametric Kolmogorov-Smirnov (KS) two-sample test in SAS (version 9.3; SAS Institute). The distributions of sensitivity classifications for orchard populations in different apple production regions (New York, Mid-Atlantic, and Midwest) were compared in an exploratory manner using $\chi^{2}$ tests in SAS (version 9.3; SAS Institute).

Specifically, the classification of orchard populations using the $\mathrm{K}-\mathrm{S}$ two-sample test was conducted by comparing distributions of trifloxystrobin sensitivity (i.e., quantitative resistance) responses for the orchard populations being evaluated ("test orchards") with both the resistant and the sensitive standards, as previously described (19). First, we determined whether or not the distribution of responses for a given test orchard was significantly different from the distribution of responses for the standard population with practical resistance $\left(\mathrm{Pr}>\mathrm{Ksa}_{\text {Resistant }}\right)$. Second, we determined whether or not the distribution of responses for a given test orchard was significantly different from the distribution of responses for the standard population that was sensitive $\left(\mathrm{Pr}>\mathrm{Ksa}_{\text {Sensitive; }}\right.$; Table 1 ). The outcomes of the K-S tests were used in conjunction to classify the population. When the two tests used in conjunction there can be three possible outcomes: (i) the test population is significantly different from the resistant population $\left(\mathrm{Pr}>\mathrm{Ksa}_{\text {Resistant }}<\right.$ 0.05 ) but not the sensitive population ( $\mathrm{Pr}>\mathrm{Ksa}_{\text {Sensitive }}>0.05$ ), (ii) the test population is significantly different from the sensitive population $\left(\mathrm{Pr}>\mathrm{Ksa}_{\text {Sensitive }}<0.05\right)$ but not the resistance population ( $\mathrm{Pr}>\mathrm{Ksa}_{\text {Resistant }}>0.05$ ), or (iii) the test population is significantly different from both population standards $\left(\mathrm{Pr}>\mathrm{Ksa}_{\text {Resistant }}<\right.$ 0.05 and $\mathrm{Pr}>\mathrm{Ksa}_{\text {Sensitive }}<0.05$ ). In outcomes $\mathrm{i}$ and ii, classification is simple. If the distribution of test population is not significantly different from the sensitive or resistant population, it is resistant or sensitive respectively. However, outcome iii represents the possibility that the distribution of responses from the test orchard can be significantly more sensitive or more resistant than the standard or be intermediate to both standards. In the case of outcome iii, one would use a visual depiction of responses such as a histogram for the test population and two standards to determine whether or not the test orchard was more or less sensitive or fell in the between the two standards. To simplify the classification for outcome iii, the population mean \%RG for the test and standard populations was used. Presented are a series of examples for the classification of orchards in outcome iii. If the test population mean \% RG was greater than that of the resistant standard, then the test orchard is significantly more resistant than the resistant standard. Similarly, if the test population mean \%RG was lower than that of the sensitive standard, then the test orchard is significantly more sensitive than the sensitive standards. Finally, if the test population mean \% RG was intermediate to that of the sensitive and resistant standard, the orchard has classified as being reduced sensitive (Table 1).

Determination of QoI qualitative resistance in orchard populations of $\boldsymbol{V}$. inaequalis. Because practical resistance can also 
Table 1. Practical resistance classification, number of isolates, percent relative growth, sensitivity, number of isolates with G143A resistance, year collected, and location from which Venturia inaequalis populations were evaluated for trifloxystrobin resistance

\begin{tabular}{|c|c|c|c|c|c|c|c|c|}
\hline Desig. ${ }^{a}$ & Classification $^{\mathbf{b}}$ & $n$ & RG $(\%)^{\mathbf{c}}$ & $\begin{array}{c}\text { Pr }> \\
\text { Ksa }_{\text {Resistant }}{ }^{d}\end{array}$ & $\begin{array}{c}\text { Pr }> \\
\text { Ksa }_{\text {Sensitive }} \text { d }\end{array}$ & $\begin{array}{c}\text { Number with } \\
\text { G143A }^{\mathbf{e}}\end{array}$ & Year & State \\
\hline 1 & Sensitive & 32 & $0.0 \pm 0.0$ & $<0.0001$ & $<0.0001$ & 0 & 2009 & New York \\
\hline 2 & Sensitive & 30 & $19.8 \pm 4.7$ & 0.0125 & 0.3876 & 0 & 2007 & New Hampshire \\
\hline 3 & Sensitive & 27 & $14.5 \pm 8.0$ & $<0.0001$ & 0.006 & 0 & 2007 & Massachusetts \\
\hline 4 & Sensitive & 30 & $12.5 \pm 6.4$ & $<0.0001$ & 0.0072 & 0 & 2007 & Rhode Island \\
\hline 5 & Sensitive & 30 & $0.5 \pm 0.5$ & $<0.0001$ & $<0.0001$ & 0 & 2007 & New York \\
\hline $5 \mathrm{a}$ & Sensitive & 26 & $10.4 \pm 3.6$ & $<0.0001$ & 0.0101 & 4 & 2011 & New York \\
\hline 6 & Sensitive & 31 & $12.8 \pm 4.1$ & 0.0002 & 0.0566 & 0 & 2005 & Massachusetts \\
\hline $6 \mathrm{a}$ & Sensitive & 29 & $14.6 \pm 4.6$ & 0.0002 & 0.1618 & 0 & 2006 & Massachusetts \\
\hline 7 & Sensitive & 30 & $23.2 \pm 5.2$ & 0.0242 & 0.586 & 0 & 2006 & Vermont \\
\hline 8 & Sensitive & 16 & $7.6 \pm 4.5$ & 0.0002 & 0.018 & 0 & 2007 & Maine \\
\hline 9 & Sensitive & 30 & $26.1 \pm 3.5$ & 0.0002 & 0.3876 & 0 & 2005 & Massachusetts \\
\hline 10 & Sensitive & 30 & $2.8 \pm 2.8$ & $<0.0001$ & 0.0001 & 0 & 2007 & New York \\
\hline 11 & Sensitive & 30 & $17.9 \pm 4.3$ & 0.0015 & 0.586 & 0 & 2007 & Maine \\
\hline 12 & Sensitive & 30 & $29.2 \pm 4.0$ & 0.0033 & 0.799 & 0 & 2005 & New York \\
\hline $12 \mathrm{a}$ & Sensitive & 30 & $18.6 \pm 5.6$ & 0.0005 & 0.3876 & 0 & 2006 & New York \\
\hline $12 \mathrm{~b}$ & Sensitive & 30 & $18.6 \pm 3.6$ & 0.0007 & 0.2365 & 0 & 2007 & New York \\
\hline $12 \mathrm{c}$ & Sensitive & 32 & $6.3 \pm 3.6$ & $<0.0001$ & 0.0009 & 3 & 2009 & New York \\
\hline $12 \mathrm{~d}$ & Sensitive & 50 & $12.7 \pm 4.3$ & $<0.0001$ & 0.0007 & 7 & 2008 & New York \\
\hline 13 & Sensitive & 30 & $11.3 \pm 2.7$ & $<0.0001$ & 0.0165 & 0 & 2004 & New York \\
\hline $13 \mathrm{a}$ & Sensitive & 30 & $1.67 \pm 1.62$ & 0.0004 & 0.586 & 0 & 2005 & New York \\
\hline $13 b$ & Sensitive & 32 & $0.0 \pm 0.0$ & $<0.0001$ & $<0.0001$ & 0 & 2006 & New York \\
\hline $13 \mathrm{c}$ & Sensitive & 30 & $3.0 \pm 1.8$ & $<0.0001$ & 0.0004 & 0 & 2007 & New York \\
\hline $13 \mathrm{~d}$ & Sensitive & 34 & $0.9 \pm 0.5$ & $<0.0001$ & $<0.0001$ & 0 & 2008 & New York \\
\hline $13 \mathrm{e}$ & Sensitive & 22 & $0.1 \pm 0.1$ & $<0.0001$ & 0.0003 & 0 & 2010 & New York \\
\hline $13 \mathrm{f}$ & Sensitive & 25 & $4.51 \pm 2.7$ & $<0.0001$ & 0.0021 & 0 & 2011 & New York \\
\hline 14 & Sensitive & 36 & $8.2 \pm 2.2$ & $<0.0001$ & 0.0008 & 0 & 2008 & Rhode Island \\
\hline 15 & Sensitive & 24 & $33.6 \pm 5.2$ & 0.0222 & 0.1379 & 0 & 2007 & Massachusetts \\
\hline 16 & Sensitive & 30 & $2.2 \pm 1.7$ & $<0.0001$ & 0.0001 & 0 & 2007 & New Hampshire \\
\hline 17 & Sensitive & 19 & $35.5 \pm 3.4$ & 0.003 & 0.1207 & 0 & 2007 & New York \\
\hline 18 & Sensitive & 30 & $27.6 \pm 5.3$ & 0.0366 & 0.9525 & 0 & 2005 & New York \\
\hline $18 \mathrm{a}$ & Sensitive & 31 & $4.1 \pm 2.0$ & $<0.0001$ & 0.0023 & 0 & 2006 & New York \\
\hline $18 \mathrm{~b}$ & Sensitive & 30 & $15.5 \pm 3.6$ & 0.0002 & 0.0354 & 0 & 2007 & New York \\
\hline $18 \mathrm{c}$ & Sensitive & 32 & $0.5 \pm 0.5$ & $<0.0001$ & $<0.0001$ & 0 & 2009 & New York \\
\hline 19 & Sensitive & 21 & $10.6 \pm 3.2$ & 0.0002 & 0.0114 & 0 & 2008 & Rhode Island \\
\hline 20 & Sensitive & 30 & $34.8 \pm 4.9$ & 0.0366 & 0.2365 & 0 & 2007 & Rhode Island \\
\hline 21 & Sensitive & 30 & $20.6 \pm 3.3$ & $<0.0001$ & 0.1344 & 0 & 2006 & Massachusetts \\
\hline 22 & Sensitive & 32 & $4.4 \pm 3.1$ & $<0.0001$ & 0.0003 & 0 & 2009 & Maine \\
\hline 23 & Sensitive & 30 & $12.2 \pm 3.3$ & 0.0005 & 0.0354 & 0 & 2007 & Rhode Island \\
\hline $23 \mathrm{a}$ & Sensitive & 15 & $0.0 \pm 0.0$ & $<0.0001$ & 0.0015 & 0 & 2009 & Rhode Island \\
\hline 24 & Sensitive & 30 & $12.1 \pm 5.4$ & $<0.0001$ & 0.0165 & 0 & 2006 & New York \\
\hline 25 & Sensitive & 22 & $25.13 \pm 6.3$ & 0.0674 & 0.5995 & 0 & 2009 & Michigan \\
\hline 26 & Sensitive & 47 & $20.7 \pm 3.5$ & 0.0012 & 0.4843 & 0 & 2008 & West Virginia \\
\hline $26 \mathrm{a}$ & Sensitive & 39 & $18.1 \pm 6.4$ & $<0.0001$ & 0.0177 & 0 & 2010 & West Virginia \\
\hline 27 & Sensitive & 30 & $33.1 \pm 7.0$ & 0.0242 & 0.0713 & 0 & 2006 & New York \\
\hline 28 & Sensitive & 33 & $7.8 \pm 3.6$ & $<0.0001$ & 0.0036 & 0 & 2009 & New York \\
\hline 29 & Sensitive & 30 & $5.0 \pm 3.7$ & 0.0354 & 0.004 & 0 & 2005 & New York \\
\hline $29 a$ & Sensitive & 30 & $5.1 \pm 3.7$ & $<0.0001$ & 0.0004 & 0 & 2006 & New York \\
\hline 30 & Sensitive & 30 & $2.3 \pm 1.2$ & $<0.0001$ & 0.0001 & 0 & 2007 & West Virginia \\
\hline 31 & Sensitive & 30 & $33.0 \pm 5.1$ & 0.0657 & 0.2365 & 0 & 2005 & New York \\
\hline 32 & Sensitive & 30 & $5.9 \pm 2.8$ & $<0.0001$ & 0.0029 & 0 & 2005 & Massachusetts \\
\hline $32 \mathrm{a}$ & Sensitive & 30 & $7.6 \pm 2.9$ & $<0.0001$ & 0.0165 & 0 & 2006 & Massachusetts \\
\hline 33 & Sensitive & 30 & $25.9 \pm 4.4$ & 0.0025 & 1 & $\mathbf{0}$ & 2005 & New York \\
\hline 34 & Sensitive & 36 & $0.1 \pm 0.1$ & $<0.0001$ & $<0.0001$ & 0 & 2009 & New York \\
\hline $34 \mathrm{a}$ & Sensitive & 16 & $5.5 \pm 5.1$ & $<0.0001$ & 0.0068 & 0 & 2011 & New York \\
\hline 35 & Sensitive & 30 & $16.2 \pm 4.2$ & 0.0005 & 0.1344 & 0 & 2006 & New York \\
\hline 36 & Sensitive & 22 & $32.7 \pm 5.3$ & 0.0263 & 0.7232 & 0 & 2007 & New York \\
\hline 37 & Sensitive & 30 & $36.3 \pm 4.3$ & 0.0056 & 0.1494 & 0 & 2007 & Massachusetts \\
\hline & & & & & & & \multicolumn{2}{|c|}{ (continued on next page } \\
\hline
\end{tabular}

${ }^{a}$ Designation. Orchard populations that were reexamined additional times in later years are denoted by the presence of letters (e.g., 18, 18a, 18b, and so on). Rows highlighted in bold represent sensitive and resistance standard orchard populations.

${ }^{b}$ Orchard classification in terms of practical resistance status was determined in the study by comparing the distribution of quantitative quinone-outsideinhibitor (QoI) fungicide sensitivity responses (isolate percent mean relative growth values on analytical-grade trifloxystrobin at $0.02 \mu \mathrm{g} / \mathrm{ml}$ ) for a given $V$. inaequalis orchard population with standard orchard populations with and without practical resistance to QoI fungicides resistant using a KolmogorovSmirnov two-sample test.

${ }^{c}$ Quantitative resistance responses for orchard population. Quantitative (polygenic or partial) resistance response result from multiple unknown factors imparting a minor level of fungicide insensitivity outside of the context of cytochrome $b(c y t b)$ gene mutation. This response is measured by the mean percent growth of $V$. inaequalis isolates on medium amended with analytical-grade trifloxystrobin at $0.02 \mu \mathrm{g} / \mathrm{ml}$ relative to that on nonfungicide medium (\%RG). Values are means and standard errors of $n$ isolates for each population, with five single-conidium colonies for each isolate.

${ }^{\mathrm{d}}$ Probability that the distribution of responses for a given test orchard was significantly different from the distribution of responses for the standard orchard population with $\left(\mathrm{Pr}>\mathrm{Ksa}_{\text {Resistant }}\right)$ or without $\left(\mathrm{Pr}>\mathrm{Ksa}_{\text {Sensitive }}\right)$ practical resistance to trifloxystrobin. Ksa is the test statistic for the Kolmogorov-Smirnov two-sample test. See the Materials and Methods section for an explanation of orchard classification using both probabilities.

${ }^{\mathrm{e}}$ Number of isolates in a population with qualitative resistance, which is a vertical or complete resistance response whereby isolates are highly resistant to QoI fungicides resulting from a cyt $b$ gene mutation. 
Table 1. (continued from preceding page)

\begin{tabular}{|c|c|c|c|c|c|c|c|c|}
\hline Desig. ${ }^{a}$ & Classification $^{\mathbf{b}}$ & $n$ & RG $(\%)^{\mathrm{c}}$ & $\begin{array}{c}\text { Pr }> \\
\text { Ksa }_{\text {Resistant }}{ }^{d}\end{array}$ & $\begin{array}{c}\text { Pr }> \\
\text { Ksa }_{\text {Sensitive }}{ }^{\mathrm{d}}\end{array}$ & $\begin{array}{c}\text { Number with } \\
\text { G143A }^{\mathbf{e}}\end{array}$ & Year & State \\
\hline 38 & Sensitive & 19 & $3.8 \pm 3.9$ & $<0.0001$ & 0.0019 & 0 & 2005 & New York \\
\hline 39 & Sensitive & 30 & $34.0 \pm 7.4$ & 0.0543 & 0.0713 & 0 & 2005 & West Virginia \\
\hline $39 a$ & Sensitive & 30 & $11.3 \pm 4.1$ & $<0.0001$ & 0.0204 & 0 & 2006 & West Virginia \\
\hline 40 & Sensitive & 16 & $18.0 \pm 3.9$ & 0.003 & 0.616 & 0 & 2004 & New York \\
\hline 41 & Sensitive & 30 & $20.5 \pm 3.8$ & 0.011 & 0.0713 & 0 & 2006 & West Virginia \\
\hline 42 & Sensitive & 30 & $17.0 \pm 6.5$ & $<0.0001$ & 0.1344 & 0 & 2005 & New York \\
\hline 43 & Sensitive & 30 & $2.7 \pm 1.7$ & $<0.0001$ & 0.0001 & 0 & 2006 & Vermont \\
\hline 44 & Sensitive & 31 & $8.3 \pm 3.8$ & $<0.0001$ & 0.0023 & 0 & 2005 & West Virginia \\
\hline 45 & Sensitive & 30 & $28.9 \pm 6.9$ & 0.0657 & 0.586 & 0 & 2005 & New York \\
\hline 46 & Sensitive & 30 & $12.7 \pm 3.9$ & 0.0004 & 0.0354 & 0 & 2005 & Ohio \\
\hline 47 & Sensitive & 30 & $25.4 \pm 9.9$ & $<0.0001$ & 0.0713 & 0 & 2007 & New York \\
\hline 48 & Sensitive & 30 & $16.4 \pm 4.4$ & 0.0037 & 0.1344 & 0 & 2007 & Rhode Island \\
\hline 49 & Sensitive & 27 & $0.5 \pm 0.4$ & $<0.0001$ & $<0.0001$ & 0 & 2009 & Rhode Island \\
\hline 50 & Sensitive & 30 & $14.0 \pm 3.2$ & $<0.0001$ & 0.0354 & 0 & 2005 & Ohio \\
\hline 51 & Sensitive & 30 & $0.00 \pm 0.0$ & $<0.0001$ & $<0.0001$ & 0 & 2009 & New York \\
\hline 52 & Sensitive & 30 & $11.2 \pm 4.2$ & $<0.0001$ & 0.0165 & 0 & 2005 & New Hampshire \\
\hline 53 & Sensitive & 27 & $4.4 \pm 4.4$ & $<0.0001$ & 0.0005 & 0 & 2008 & Pennsylvania \\
\hline 54 & Sensitive & 34 & $0.1 \pm 0.1$ & $<0.0001$ & $<0.0001$ & 0 & 2009 & Indiana \\
\hline 55 & Sensitive & 20 & $0.0 \pm 0.0$ & $<0.0001$ & 0.0004 & 0 & 2009 & Maine \\
\hline 56 & Sensitive & 26 & $19.6 \pm 8.1$ & 0.0072 & 0.1849 & 0 & 2006 & Massachusetts \\
\hline 57 & Sensitive & 30 & $8.4 \pm 3.0$ & $<0.0001$ & 0.0165 & 0 & 2005 & West Virginia \\
\hline 58 & Sensitive & 30 & $6.0 \pm 2.4$ & $<0.0001$ & 0.0029 & 0 & 2006 & New York \\
\hline 59 & Sensitive & 30 & $5.3 \pm 2.2$ & $<0.0001$ & $<0.0001$ & 0 & 2007 & New York \\
\hline $59 a$ & Sensitive & 34 & $0.6 \pm 0.3$ & $<0.0001$ & $<0.0001$ & 0 & 2008 & New York \\
\hline $59 b$ & Sensitive & 33 & $0.3 \pm 0.3$ & $<0.0001$ & 0.0003 & 0 & 2009 & New York \\
\hline 60 & Sensitive & 30 & $27.7 \pm 4.7$ & 0.0061 & 0.586 & 0 & 2007 & Massachusetts \\
\hline $60 \mathrm{a}$ & Sensitive & 34 & $3.4 \pm 1.6$ & $<0.0001$ & 0.0001 & 0 & 2008 & Massachusetts \\
\hline 61 & Sensitive & 27 & $12.6 \pm 0.3$ & $<0.0001$ & 0.0448 & 0 & 2008 & Rhode Island \\
\hline 62 & Sensitive & 29 & $0.00 \pm 0.0$ & $<0.0001$ & $<0.0001$ & 0 & 2009 & Maine \\
\hline 63 & Sensitive & 32 & $28.1 \pm 4.0$ & 0.0035 & 0.4994 & 0 & 2005 & Ohio \\
\hline 64 & Sensitive & 30 & $8.5 \pm 3.7$ & $<0.0001$ & 0.0165 & 0 & 2005 & New York \\
\hline $64 a$ & Sensitive & 30 & $3.7 \pm 2.3$ & $<0.0001$ & 0.0008 & 0 & 2006 & New York \\
\hline $64 b$ & Sensitive & 30 & $4.1 \pm 2.4$ & $<0.0001$ & 0.0011 & 0 & 2007 & New York \\
\hline 65 & Sensitive & 41 & $1.6 \pm 1.6$ & $<0.0001$ & $<0.0001$ & 0 & 2009 & Michigan \\
\hline 66 & Sensitive & 30 & $15.0 \pm 4.4$ & $<0.0001$ & 0.0703 & 0 & 2007 & Rhode Island \\
\hline 67 & Sensitive & 30 & $5.0 \pm 2.2$ & $<0.0001$ & 0.0029 & 0 & 2006 & New York \\
\hline 68 & Sensitive & 24 & $20.2 \pm 4.6$ & 0.0013 & 0.2348 & 0 & 2005 & Massachusetts \\
\hline 69 & Sensitive & 20 & $20.7 \pm 6.5$ & 0.024 & 0.2904 & 0 & 2006 & Massachusetts \\
\hline 70 & Sensitive & 33 & $8.5 \pm 3.1$ & $<0.0001$ & 0.0096 & 0 & 2009 & New York \\
\hline 71 & Sensitive & 29 & $35.5 \pm 7.3$ & 0.0145 & 0.2775 & 0 & 2007 & New York \\
\hline 72 & Sensitive & 30 & $34.0 \pm 3.7$ & 0.0048 & 0.2365 & 0 & 2005 & West Virginia \\
\hline 73 & Sensitive & 30 & $23.5 \pm 4.6$ & 0.0048 & 0.799 & 0 & 2005 & West Virginia \\
\hline 74 & Sensitive & 29 & $23.0 \pm 3.9$ & 0.0006 & 0.8397 & 0 & 2006 & Rhode Island \\
\hline 75 & Sensitive & 30 & $31.7 \pm 4.9$ & 0.0269 & 0.9525 & 0 & 2006 & Massachusetts \\
\hline 76 & Sensitive & 30 & $31.8 \pm 9.1$ & 0.0598 & 0.586 & 0 & 2005 & Wisconsin \\
\hline 77 & Sensitive & 33 & $19.9 \pm 3.1$ & $<0.0001$ & 0.1737 & 0 & 2004 & New York \\
\hline 78 & Sensitive & 21 & $18.6 \pm 5.3$ & 0.0164 & 0.7232 & 0 & 2010 & Maine \\
\hline 79 & Sensitive & 26 & $17.86 \pm 4.9$ & 0.0052 & 0.3752 & 1 & 2011 & Maine \\
\hline 80 & Sensitive & 25 & $0.316 \pm 0.31$ & $<0.0001$ & $<0.0001$ & 1 & 2011 & Vermont \\
\hline 81 & Sensitive & 24 & $32.70 \pm 3.8$ & 0.0133 & 0.2656 & 1 & 2011 & Maine \\
\hline 82 & Sensitive & 38 & $11.1 \pm 4.0$ & $<0.0001$ & 0.0013 & 4 & 2008 & Rhode Island \\
\hline 83 & Sensitive & 16 & $2.39 \pm 2$ & $<0.0001$ & 0.0048 & 2 & 2011 & North Carolina \\
\hline $31 \mathrm{a}$ & Resistant & 17 & $68.7 \pm 13.0$ & 0.2329 & 0.003 & 8 & 2008 & New York \\
\hline $33 \mathrm{a}$ & Resistant & 30 & $76.7 \pm 3.9$ & 0.0002 & $<0.0001$ & 0 & 2007 & New York \\
\hline $48 \mathrm{a}$ & Resistant & 31 & $28.8 \pm 4.9$ & 0.0125 & 0.9525 & 10 & 2008 & Rhode Island \\
\hline $62 \mathrm{a}$ & Resistant & 24 & $12.03 \pm 5.8$ & $<0.0001$ & 0.0134 & 8 & 2011 & Maine \\
\hline $81 \mathrm{a}$ & Resistant & 30 & $46.7 \pm 4.0$ & 0.1333 & 0.0354 & 0 & 2007 & Vermont \\
\hline $81 \mathrm{~b}$ & Resistant & 35 & $25.6 \pm 6.1$ & 0.0012 & 0.3934 & 10 & 2008 & Vermont \\
\hline 84 & Resistant & 30 & $67.6 \pm 4.9$ & 0.0088 & $<0.0001$ & 0 & 2005 & New York \\
\hline 85 & Resistant & 31 & $75.6 \pm 3.3$ & 0.0001 & $<0.0001$ & 0 & 2005 & New York \\
\hline 86 & Resistant & 30 & $43.2 \pm 7.1$ & 0.8005 & 0.0165 & 0 & 2006 & New York \\
\hline 87 & Resistant & 30 & $44.2 \pm 6.1$ & 0.1225 & 0.0129 & 0 & 2007 & New York \\
\hline 88 & Resistant & 30 & $50.1 \pm 5.2$ & 0.1032 & 0.0165 & 0 & 2007 & New Hampshire \\
\hline 89 & Resistant & 30 & $51.8 \pm 8.6$ & 0.8005 & 0.0029 & 0 & 2006 & New York \\
\hline 90 & Resistant & 30 & $71.0 \pm 5.1$ & 0.0033 & $<0.0001$ & 0 & 2007 & New York \\
\hline 91 & Resistant & 31 & $44.1 \pm 5.5$ & 0.6083 & 0.0855 & 0 & 2005 & Ohio \\
\hline 92 & Resistant & 30 & $51.6 \pm 5.3$ & 0.2145 & 0.0165 & 0 & 2007 & Rhode Island \\
\hline 93 & Resistant & 30 & $54.5 \pm 3.9$ & 0.0217 & 0.0072 & 0 & 2007 & New York \\
\hline 94 & Resistant & 30 & $45.0 \pm 4.1$ & 0.0543 & 0.0165 & 0 & 2007 & Connecticut \\
\hline 95 & Resistant & 30 & $57.1 \pm 7.8$ & 0.1988 & 0.0011 & 0 & 2006 & New York \\
\hline 96 & Resistant & 30 & $67.9 \pm 5.4$ & 0.0111 & $<0.0001$ & 0 & 2005 & New York \\
\hline 97 & Resistant & 31 & $41.0 \pm 4.1$ & 0.0493 & 0.1344 & 0 & 2005 & Ohio \\
\hline & & & & & & & \multicolumn{2}{|c|}{ (continued on next page } \\
\hline
\end{tabular}


occur solely on the basis of isolates with qualitative or vertical responses resulting from $c y t b$ gene mutations, both qualitative and quantitative resistance phenomena need to be considered. The prevalence of QoI qualitative resistance was also investigated for each orchard population of $V$. inaequalis. To determine presence of qualitative resistance in individual isolates, an in vitro germination assay and polymerase chain reaction (PCR) restriction fragment length polymorphism (RFLP) analysis were used. All single-lesion conidial isolates from each of the orchard populations sampled were subjected to the in vitro germination assay by placing $200 \mu \mathrm{l}$ of the conidial suspension (see above) on PDA (Difco Laboratories) amended with trifloxystrobin $(0.2 \mu \mathrm{g} / \mathrm{ml})$ to suppress quantitative resistance response, and amended with salicylhydroxamic acid (SHAM; $100 \mu \mathrm{g} / \mathrm{ml}$, analytical standard; Sigma-Aldrich) to inhibit the alternative respiratory pathway, as suggested by Köller et al. (18). The combination of SHAM with a high dose QoI fungicide such as trifloxystrobin induces a synergistic mycelial growth inhibition that effectively eliminates quantitative QoI resistance responses from masking the effects of $c y t b$ mutations $(9,29)$. Plates were incubated for 14 days at 23 to $25^{\circ} \mathrm{C}$ and evaluated for the growth of $V$. inaequalis colonies (radial growth $>1 \mathrm{~mm}$ in diameter), which indicates the presence of qualitative QoI resistance for that isolate (18). In addition to the evaluation of the quantitative resistance response as described above, populations were also considered to have practical resistance to QoI fungicides when more than $25 \%$ of the population members possessed qualitative resistance, as previously suggested (20).

To confirm the molecular basis of qualitative resistance for a subset (33 to $100 \%$ ) of $V$. inaequalis isolates from each population, PCR-RFLP was performed as described by Fontaine et al. (10), with several modifications. Prior to DNA extraction, colonies were grown from a single germinating conidium on trifloxystrobinamended media $(0.02 \mu \mathrm{g} / \mathrm{ml})$ for 5 weeks at 23 to $25^{\circ} \mathrm{C}$. Isolation of $V$. inaequalis tissue for DNA extraction was accomplished by gently scraping mycelium from the surface of the medium using a scalpel. Mycelia from each isolate $(100 \mathrm{mg})$ were ground using liquid nitrogen and total DNA was extracted using an Omega BioTek Plant DNA extraction kit (Omega Bio-Tek) following the manufacturer's protocol. To determine the presence of the $c y t b$ G143A mutation, PCR was performed to amplify a 950-bp am- plicon from the cyt $b$ gene using $V$. inaequalis-specific primers ViCytB-5F (5'-GGACCAAGTAATCACT GGTGTATGG-3') and ViCytB-6071R (5'-TTGAAAGCTAGGCTAGGGCGAACA-3') (7). PCR reactions were made in $25-\mu$ reaction volumes and contained $1 \times$ PCR buffer, $0.4 \mu \mathrm{M}$ each primer, $2.5 \mathrm{mM} \mathrm{MgCl}_{2}, 200$ $\mu \mathrm{M}$ each dNTP, $0.625 \mathrm{U}$ of GoTaq Flexi DNA polymerase (Promega Corporation), and $1 \mu \mathrm{l}$ of extracted DNA. This PCR reaction was performed using an iCycler thermal cycler (Bio-Rad Laboratories) with the following cycling protocol: initial denaturation for $3 \mathrm{~min}$ at $94^{\circ} \mathrm{C} ; 30$ cycles consisting of $30 \mathrm{~s}$ at $94^{\circ} \mathrm{C}, 30 \mathrm{~s}$ at $53^{\circ} \mathrm{C}$, and $30 \mathrm{~s}$ at $72^{\circ} \mathrm{C}$; and a final extension step of $5 \mathrm{~min}$ at $72^{\circ} \mathrm{C}$. PCR products were then separated on a $1.0 \%$ agarose gel in $1 \times$ TAE buffer (44.5 mM Tris-borate and $1 \mathrm{mM}$ EDTA, $\mathrm{pH}$ 8.0) for $45 \mathrm{~min}$ at $100 \mathrm{~V}$ and imaged on a KODAK Gel Logic 200 Imaging System (Eastman Kodak Company). RFLP analysis was performed on PCR products using $5 \mathrm{U}$ of Fnu4HI restriction enzyme (New England Biolabs) on $0.5 \mu \mathrm{g}$ of DNA from the PCR product. The reaction was incubated at $37^{\circ} \mathrm{C}$ for $12 \mathrm{~h}$. After incubating, a final inactivation step of $25 \mathrm{~min}$ at $65^{\circ} \mathrm{C}$ was performed with an iCycler thermal cycler (Bio-Rad Laboratories) to inactivate the Fnu4HI restriction, as required by the manufacturer's protocol, and DNA fragments were analyzed by electrophoresis on a $1.0 \%$ agarose gel (Invitrogen).

Stability of QoI qualitative resistance in isolates of $V$. inaequalis in the absence of QoI-induced selective pressure. A subset of 28 isolates from orchards surveyed in 2010 and 2011 with a range of quantitative resistant phenotypes was evaluated for the presence and stability of the $c y t b$ G143A mutation (Table 2). To establish the presence of the $c y t b$ G143A mutation, isolates were subjected to the in vitro germination assay, PCR, and RFLP analysis described above. To test for stability of the mutation within isolates, colonies were first grown on PDA amended with trifloxystrobin and SHAM (PDA-TSHAM), transferred to medium amended with trifloxystrobin $(0.02 \mu \mathrm{g} / \mathrm{ml})$, and allowed to grow for 5 weeks at 23 to $25^{\circ} \mathrm{C}$ to maintain selective pressure. Once $V$. inaequalis colonies were 5 weeks old, two agar plugs were removed from the periphery of the colony with a 5-mm-diameter cork borer and transferred to new trifloxystrobin-amended medium and nonamended PDA. At each of six successive 5-week time points, each isolate was transferred to either fresh PDA or fresh

Table 1. (continued from preceding page)

\begin{tabular}{|c|c|c|c|c|c|c|c|c|}
\hline Desig. $^{a}$ & Classification $^{\text {b }}$ & $n$ & RG $(\%)^{\mathrm{c}}$ & $\begin{array}{c}\text { Pr }> \\
\text { Ksa }_{\text {Resistant }}{ }^{d}\end{array}$ & $\begin{array}{c}\operatorname{Pr}> \\
\text { Ksa }_{\text {Sensitive }}{ }^{d}\end{array}$ & $\begin{array}{c}\text { Number with } \\
\text { G143A }^{\mathbf{e}}\end{array}$ & Year & State \\
\hline 98 & Resistant & 30 & $49.7 \pm 6.9$ & 0.2145 & 0.0713 & 0 & 2007 & Massachusetts \\
\hline 99 & Resistant & 30 & $78.8 \pm 5.4$ & 0.017 & $<0.0001$ & 0 & 2005 & New York \\
\hline 100 & Resistant & 30 & $45.2 \pm 3.0$ & 0.0147 & 0.0032 & 0 & 2007 & Rhode Island \\
\hline 101 & Resistant & 30 & $48.4 \pm 5.5$ & 0.1032 & 0.0072 & 0 & 2004 & New York \\
\hline 102 & Resistant & 30 & $47.4 \pm 6.9$ & 0.4249 & 0.0354 & 0 & 2007 & New York \\
\hline 103 & Resistant & 30 & $70.9 \pm 4.4$ & 0.0019 & $<0.0001$ & 0 & 2005 & New York \\
\hline 104 & Resistant & 30 & $46.3 \pm 5.3$ & 0.0269 & 0.0165 & 4 & 2007 & New York \\
\hline 105 & Resistant & 19 & $41.8 \pm 9.6$ & 0.6632 & 0.0546 & 5 & 2010 & West Virginia \\
\hline 106 & Resistant & 20 & $31.64 \pm 7$ & 0.2391 & 0.5121 & 5 & 2011 & Maine \\
\hline 107 & Resistant & 32 & $21.3 \pm 5.6$ & 0.0001 & 0.086 & 9 & 2009 & Michigan \\
\hline 108 & Resistant & 20 & $50.95 \pm 5.87$ & 0.285 & 0.0107 & 6 & 2009 & Michigan \\
\hline 109 & Resistant & 15 & $10.73 \pm 6.1$ & 0.0007 & 0.0815 & 5 & 2011 & Maine \\
\hline 110 & Resistant & 15 & $94.19 \pm 4.9$ & 0.0001 & $<0.0001$ & 6 & 2011 & Vermont \\
\hline 111 & Resistant & 24 & $19.8 \pm 27.7$ & $<0.0001$ & 0.0466 & 9 & 2011 & Maine \\
\hline 112 & Resistant & 25 & $58.4 \pm 12.7$ & 0.27 & 0.002 & 13 & 2008 & New York \\
\hline 113 & Resistant & 34 & $54.7 \pm 7.2$ & 0.1799 & 0.0033 & 21 & 2010 & New York \\
\hline 114 & Resistant & 35 & $67.7 \pm 9.4$ & 0.0002 & $<0.0001$ & 22 & 2008 & New York \\
\hline 115 & Resistant & 18 & $3.04 \pm 2.2$ & $<0.0001$ & 0.0035 & 13 & 2011 & Virginia \\
\hline 116 & Resistant & 21 & $69.92 \pm 7.2$ & 0.0449 & 0.0001 & 18 & 2011 & Massachusetts \\
\hline 117 & Resistant & 21 & $100.35 \pm 3.2$ & $<0.0001$ & $<0.0001$ & 20 & 2011 & New York \\
\hline 118 & Resistant & 37 & $49.9 \pm 2.4$ & 1 & $<0.0001$ & 11 & 2012 & New York \\
\hline $18 \mathrm{~d}$ & Reduced sensitive & 25 & $36.3 \pm 11.6$ & 0.2404 & 0.5774 & 1 & 2010 & New York \\
\hline $19 \mathrm{a}$ & Reduced sensitive & 30 & $39.8 \pm 3.0$ & 0.0088 & 0.0165 & 0 & 2007 & Rhode Island \\
\hline $30 \mathrm{a}$ & Reduced sensitive & 30 & $33.9 \pm 6.6$ & 0.0016 & 0.0308 & 0 & 2006 & West Virginia \\
\hline $35 \mathrm{a}$ & Reduced sensitive & 30 & $34.3 \pm 4.1$ & 0.0088 & 0.2365 & 0 & 2004 & New York \\
\hline 118 & Reduced sensitive & 28 & $39.1 \pm 3.6$ & 0.0118 & 0.0428 & 0 & 2006 & Massachusetts \\
\hline 119 & Reduced sensitive & 30 & $39.2 \pm 3.6$ & 0.0366 & 0.0165 & 0 & 2007 & New York \\
\hline 120 & Reduced sensitive & 30 & $35.3 \pm 4.4$ & 0.3074 & 0.1344 & 0 & 2005 & New York \\
\hline
\end{tabular}


trifloxystrobin-amended $(0.02 \mu \mathrm{g} / \mathrm{ml})$ medium to mimic the absence of selection pressure or constant selection pressure, respectively. By the end of the experiment, each isolate had undergone six transfers over the course of 6 months. At the outset and at the time of each transfer, the presence of the $c y t b \mathrm{G} 143 \mathrm{~A}$ mutation was assessed for each isolate from each medium using PCR-RFLP analysis, as described above.

Eight isolates that were not resistant to trifloxystrobin were included in the study as controls. Because these isolates would not grow on trifloxystrobin-amended media, they were first grown on nonamended PDA for 5 weeks at 23 to $25^{\circ} \mathrm{C}$, then transferred to both trifloxystrobin-amended and nonamended PDA. These isolates were also evaluated for the presence of the $c y t b$ G143A mutation from each medium using PCR-RFLP analysis at each evaluation point.

\section{Results}

Trifloxystrobin sensitivity of $\boldsymbol{V}$. inaequalis orchard populations. From 2004 to 2011, 120 individual orchard populations of $V$. inaequalis were evaluated throughout the northeastern United States (Table 1). Although 27 orchard populations were represented by fewer than 25 isolates due to loss of isolate viability during collection and processing, the majority of orchard populations were represented by 25 or more isolates (Table 1 ).

During the course of the testing efforts, there were 34 (28\%) orchard populations of $V$. inaequalis determined to have less sensitivity (i.e., practical resistance) to trifloxystrobin on the basis of statistical comparison of their quantitative resistance phenotype distributions with the known standards with or without practical resistance (Tables 1 and 3). The mean $\%$ RG of quantitative resistant orchard populations was 41.0 to $100.4 \%$, with commercial orchards from Pataskala, $\mathrm{OH}(41.0 \pm 4.1 \%)$ and Newfield, NY $(100.4 \pm 3.2 \%)$ representing the end points of the range. Of the 34 orchard populations with resistance to trifloxystrobin, 32 were commercial orchards (Table 1) with a QoI use history of approximately 2 to 3 times per season, with a total of 15 or more QoI applications. The remaining two orchard populations with practical resistance to trifloxystrobin were collected from research orchards where QoI fungicides were used over the course of the planting history (Tables 1 and 3).

Of the 120 orchard populations examined, only $6(5 \%)$ orchard populations of $V$. inaequalis were found to have reduced sensitivity. The mean \%RG for these orchard populations was 33.9 to $39.2 \%$, with a research orchard $(33.9 \pm 6.6 \%)$ and a commercial orchard $(39.2 \pm 3.6 \%)$ representing the end points of the range. Of the orchard populations that had reduced sensitivity to trifloxystrobin, five were commercial orchards with a QoI use history of approximately 2 to 3 times per season, with a total of 10 to 15 QoI applications according to pesticide application records. The remaining orchard population classified as having reduced sensitivity was a research orchard treated with QoI fungicides over the planting history (Table 3).

During the course of the study, the majority $(67 \%$; 80 of 120) of orchard populations of $V$. inaequalis were found to be sensitive to trifloxystrobin on the basis of statistical comparison (K-S test) of quantitative resistance phenotype distributions to known standards with or without practical resistance (Tables 1 and 3). The mean $\%$ RG for sensitive orchard populations was 0.0 to $36.3 \%$, with a baseline orchard $(0.0 \pm 0.0 \%)$ and a sensitive commercial orchard $(36.3 \pm 4.3 \%)$ representing the end points of the range. Of the 80 orchard populations that were sensitive to trifloxystrobin, 58 were from commercial apple orchards, 12 were from research orchards, and 10 were from baseline orchards. Baselines orchards had isolates with mean $\%$ RGs of 0.0 to $18.0 \%$, with orchards from Geneva, NY $(0.0 \pm 0.0 \%)$ and Montezuma, NY $(18.0 \pm 3.9 \%)$ representing the end points of the baseline range.

Orchard populations were pooled by orchard type and region, with Connecticut, Massachusetts, Maine, New Hampshire, Rhode Island, and Vermont in New England (48 populations); Indiana, Michigan, Ohio, and Wisconsin in the Midwest (13 populations); North Carolina, Pennsylvania, Virginia, and West Virginia in the Mid-Atlantic (13 populations); and New York (46 populations). The percent resistant populations in New York (42\%) was nearly double that in the Mid-Atlantic (23\%) and higher than that in New

Table 2. Characteristics of Venturia inaequalis single-lesion conidial isolates used for the stability of the cytochrome $b$ (cyt $b$ ) G143A mutation assay

\begin{tabular}{|c|c|c|c|c|}
\hline Isolate & Isolate classification $^{a}$ & Mean $\% \mathbf{R G}^{\mathbf{b}}$ & cyt b Genotype ${ }^{c}$ & Origin \\
\hline 11.1 .11 & Resistant & 132.65 & A143 & Montague, MA \\
\hline 11.25 .11 & Resistant & 132.65 & A143 & Montague, MA \\
\hline 5.44 .11 & Resistant & 124.23 & A143 & Newfield, NY \\
\hline 5.40 .11 & Resistant & 108.49 & A143 & Newfield, NY \\
\hline 14.4 .10 & Resistant & 108.49 & A143 & Geneva, NY \\
\hline 11.13 .11 & Resistant & 100.63 & A143 & Montague, MA \\
\hline 12.19 .10 & Resistant & 100.63 & A143 & Geneva, NY \\
\hline 6.47 .10 & Resistant & 92.81 & A143 & Newfield, NY \\
\hline 8.33 .10 & Resistant & 90.47 & A143 & Geneva, NY \\
\hline 6.40 .10 & Resistant & 90.18 & A143 & Newfield, NY \\
\hline 11.11.11 & Resistant & 83.88 & A143 & Montague, MA \\
\hline 11.45 .10 & Resistant & 83.88 & A143 & Geneva, NY \\
\hline 11.17.11 & Resistant & 72.8 & A143 & Montague, MA \\
\hline 11.20.11 & Resistant & 66.5 & A143 & Montague, MA \\
\hline 41.3 .10 & Resistant & 54.26 & A143 & Geneva, NY \\
\hline 2.7 .10 & Resistant & 51.69 & A143 & Shepherdstown, WV \\
\hline 41.11 .10 & Resistant & 48.51 & A143 & Geneva, NY \\
\hline 27.35 .10 & Resistant & 45.54 & A143 & Geneva, NY \\
\hline 28.24 .11 & Resistant & 26.49 & A143 & Buckfield, ME \\
\hline 37.22 .10 & Sensitive & 52.47 & G143 & Sanford, ME \\
\hline 11.22 .11 & Sensitive & 46.93 & G143 & Montague, MA \\
\hline 25.6 .11 & Sensitive & 46.93 & G143 & Manchester, ME \\
\hline 11.8 .11 & Sensitive & 45.54 & G143 & Montague, MA \\
\hline 28.17 .11 & Sensitive & 41.46 & G143 & Buckfield, ME \\
\hline 3.35 .10 & Baseline & 0 & G143 & Geneva, NY \\
\hline 32.11 .11 & Baseline & 0 & G143 & Geneva, NY \\
\hline 32.42 .11 & Baseline & 0 & G143 & Geneva, NY \\
\hline
\end{tabular}

a Classification of individual isolates as Resistant was determined by whether or not the isolate had more than $48 \%$ relative growth (RG) and grew on trifloxystrobin $(0.2 \mu \mathrm{g} / \mathrm{ml})$ - and SHAM $(100 \mu \mathrm{g} / \mathrm{ml})$-amended medium.

${ }^{\mathrm{b}}$ Mean percent growth of $V$. inaequalis on medium amended with analytical-grade trifloxystrobin at $0.02 \mu \mathrm{g} / \mathrm{ml} \mathrm{relative}$ to that on nonfungicide medium $(\% \mathrm{RG})$. Values are means and standard errors of five randomly selected single-conidium microcolonies for each isolate.

c Amino acid at position 143 in the $c y t b$ gene of $V$. inaequalis: A indicates resistant (A143) allele and G indicates sensitive (G143) allele. 
England and the Midwest (both 31\%). These regional differences were not significant, as evaluated by a $\chi^{2}$ analysis $(P=0.542)$. Overall, $33 \%$ of all populations were either resistant or had reduced sensitivity to trifloxystrobin.

Percentage of $\boldsymbol{V}$. inaequalis isolates with qualitative QoI resistance in orchard populations. Of the 34 orchard populations of $V$. inaequalis that were considered to have practical resistance to trifloxystrobin based on the quantitative resistance analysis, 14 also had $>25 \%$ of isolates with qualitative resistance conferred by the $c y t b$ G143A mutation. The remaining 20 orchard populations with practical resistance to trifloxystrobin had few or no isolates that possessed the $c y t b$ G143A mutation $(<18 \%)$, suggesting that other resistance mechanisms were responsible for these orchards' resistance. None of the six orchard populations that were found to have reduced sensitivity or were shifted toward trifloxystrobin resistance had any isolates with the $c y t b$ G143A mutation. Of the 80 trifloxystrobin-sensitive orchard populations, 8 did have isolates with the $c y t b$ G143A mutation (Table 1). Although there were isolates with qualitative resistance in these populations, they were not sufficiently abundant ( 2 to $14 \%$ of the isolates) to exceed the threshold of $25 \%$.

Evaluation of the stability of the G143A mutation in isolates of $\boldsymbol{V}$. inaequalis. Qualitative QoI resistance to trifloxystrobin imparted by the cyt $b$ G143A mutation as determined by growth and germination on PDA-TSHAM was maintained through six con- secutive transfers over 6 months for all 19 isolates examined (Table 2). Similarly, the presence of the G143A mutation was consistently observed by PCR-RFLP analysis for all 19 isolates after each transfer on both trifloxystrobin-amended and nonamended medium. As expected, all eight sensitive or baseline isolates failed to grow on trifloxystrobin-amended medium and did not have the $c y t$ $b$ G143A mutation, as shown by PCR-RFLP analysis on cultures grown on unamended PDA medium.

\section{Discussion}

During the course of the study, the majority of orchard populations of $V$. inaequalis were found to be sensitive to trifloxystrobin based on the quantitative resistance responses for population members. Considering the number of commercial orchard sites evaluated, there appears to be widespread sensitivity to QoI fungicides for orchard populations in New York and most of the other apple production regions in the eastern United States. At the specific locations with sensitive orchard populations, QoI fungicides are still an effective means of managing apple scab. However, such broad generalizations should be made with caution, because it is clear from the study that orchard population sensitivity is highly specific to the local operation and production practices.

Although some orchard populations were tested several times during the study, little can be said about the temporal changes in quantitative QoI sensitivity because most sites were not analyzed

Table 3. Practical resistance classification, state, orchard type, and number of orchard populations $(n)$ of Venturia inaequalis examined for practical resistance to the quinone-outside-inhibitor (QoI) fungicide trifloxystrobin

\begin{tabular}{|c|c|c|c|c|}
\hline Classification $^{a}$ & State & Orchard type $^{b}$ & $n$ & Populations by state (\%) \\
\hline \multirow[t]{12}{*}{ Resistant } & Connecticut & Commercial & 1 & 100 \\
\hline & Massachusetts & Commercial & 2 & 13 \\
\hline & Maine & Commercial & 4 & 36 \\
\hline & Michigan & Commercial & 2 & 33 \\
\hline & New Hampshire & Commercial & 1 & 25 \\
\hline & New York & Commercial & 15 & 31 \\
\hline & Ohio & Commercial & 2 & 40 \\
\hline & Rhode Island & Commercial & 3 & 23 \\
\hline & Virginia & Research & 1 & 100 \\
\hline & Vermont & Commercial & 1 & 25 \\
\hline & Vermont & Research & 1 & 25 \\
\hline & West Virginia & Commercial & 1 & 10 \\
\hline Total resistant & & & 34 & 28 \\
\hline \multirow{4}{*}{ Reduced sensitive } & Massachusetts & Commercial & 1 & 7 \\
\hline & New York & Commercial & 3 & 7 \\
\hline & Rhode Island & Commercial & 1 & 8 \\
\hline & West Virginia & Research & 1 & 11 \\
\hline Total reduced sensitive & $\ldots$ & $\ldots$ & 6 & 5 \\
\hline \multirow{20}{*}{ Sensitive } & Indiana & Baseline & 1 & 100 \\
\hline & Massachusetts & Commercial & 12 & 80 \\
\hline & Maine & Commercial & 6 & 55 \\
\hline & Maine & Research & 1 & 9 \\
\hline & Michigan & Baseline & 2 & 33 \\
\hline & Michigan & Commercial & 1 & 17 \\
\hline & Michigan & Research & 1 & 17 \\
\hline & North Carolina & Commercial & 1 & 100 \\
\hline & New Hampshire & Commercial & 3 & 75 \\
\hline & New York & Baseline & 7 & 15 \\
\hline & New York & Commercial & 14 & 29 \\
\hline & New York & Research & 7 & 15 \\
\hline & Ohio & Commercial & 2 & 40 \\
\hline & Ohio & Research & 1 & 20 \\
\hline & Pennsylvania & Research & 1 & 100 \\
\hline & Rhode Island & Commercial & 9 & 69 \\
\hline & Vermont & Commercial & 2 & 50 \\
\hline & Wisconsin & Commercial & 1 & 100 \\
\hline & West Virginia & Commercial & 7 & 70 \\
\hline & West Virginia & Research & 1 & 11 \\
\hline Total sensitive & $\ldots$ & $\ldots$ & 80 & $\ldots$ \\
\hline
\end{tabular}

a Orchard classification in terms of practical resistance status was determined in the study by comparing the distribution of quantitative QoI fungicide sensitivity responses (isolate percent mean relative growth values on analytical-grade trifloxystrobin at $0.02 \mu \mathrm{g} / \mathrm{ml}$ ) for a given $V$. inaequalis orchard population with standard orchard populations with and without practical resistance to QoI fungicides resistant using a Kolmogorov-Smirnov two-sample test.

${ }^{\mathrm{b}}$ Orchard type is defined by whether the orchard was used for commercial production, used for university research, or was a baseline orchard planting. 
every year. For the 21 orchard populations that were tested in more than 1 year, 13 remained in the same QoI sensitivity classification but 4 orchard populations changed classification to resistant and 4 changed to sensitive. Fungicide application records collected for the study indicate that QoI fungicides were used in the orchards where resistance classification changed from sensitive to resistant. However, the application records also indicate QoI use in the 4 orchards where the classifications changed from resistant to sensitive and in the 18 orchards that had a consistent sensitivity classification. It is important to note that members with qualitative resistance were not absent in the four populations that were classified as sensitive when tested at a later date. Although the orchard population changes are interesting observations, it would be erroneous to make any generalizations regarding QoI use and temporal development of resistance without formal experimentation.

As with similar studies, the present study focused on many populations of $V$. inaequalis from commercial orchards $(9,10,13,18$, 20,29). A unique factor in the current study was the evaluation of a large number of baseline orchard populations (8\%; 10 of 120) that had never been exposed to a QoI fungicide or any other sitespecific chemistry. None of the isolates in the baseline orchard populations had the cyt $b$ G143A mutation but did vary in quantitative resistance response. Under the current sampling protocol, we cannot rule out the possibility that the mutation could be found in populations that are unexposed to QoI fungicides. Although we do not mean to suggest that the QoI, or any other fungicide class, is inherently mutagenic, the lack of mutation in baseline populations does provide indirect evidence supporting one of the hypotheses of van den Bosch et al. (32), who suggested that stresses induced by fungicide applications may predispose population members to more rapidly develop mutations that serve as the initiation point for selection of fungicide resistance.

A few of the orchard populations of $V$. inaequalis were classified as possessing reduced sensitivity to trifloxystrobin or shifting toward quantitative practical resistance, indicating that they should respond similarly to the resistant standard population when treated with trifloxystrobin. The distributions of these orchards were significantly different from both the sensitive and resistant standards, and their \% RG values typically fell in the range of both sensitive and resistant distributions. One explanation for these odd distributions is that the populations were primarily composed of isolates with quantitative resistance and most of the populations didn't have any isolates with qualitative resistance, which usually confers a high degree of resistance to the isolates in which it occurs $(20,31)$. Instead, a population with reduced sensitivity may be composed of individuals with resistance resulting from a myriad of unknown genetic factors working additively or synergistically to confer a range of fungicide sensitivity phenotypes (18). To date, little is known about the molecular basis of quantitative resistance to QoI fungicides in $V$. inaequalis or other pathosystems. Indeed, quantitative resistance to QoI fungicides has not been reported in any other pathosystem to date. In the six orchard populations with reduced sensitivity, there were several isolates with high \% RG values but none of the isolates possessed the $c y t b$ G143A mutation (Table 1).

We conclude from this study that populations of $V$. inaequalis with practical resistance to QoI fungicides are present in commercial apple plantings in the northeastern United States but not in the majority of orchard populations examined. Of the 40 QoI-resistant $V$. inaequalis populations, 17 had more than $25 \%$ of the isolates with the G143A mutation. In those cases, qualitative QoI resistance was likely responsible for the observed QoI-resistant phenotypes, which is in agreement with a previous study in Michigan (20). Köller et al. (18) suggested that QoI resistance occurs in two phases in orchard populations of $V$. inaequalis, beginning with occurrence of quantitative resistance that is later succeeded by development of the cyt $b$ G143A mutation that confers a high degree of qualitative resistance. We found that, in 22 of the $40 \mathrm{~V}$. inaequalis orchard populations that were classified as resistant to QoI fungicides, none of the isolates possessed the cyt $b$ G143A mutation (Table 1). These 22 orchard populations comprising quantitatively resistant isolates may be in the first phase of this process while the 18 orchards with qualitatively resistant isolates mentioned above may be in the second phase. Given that there is only a minor temporal aspect to the study, these results do not provide evidence for the two-phase development of QoI resistance in orchard populations, as previously proposed (18). However, our results do support the idea that genetic factors other than the $c y t b$ G143A mutation are involved in QoI resistance. Interestingly, the factors do not include the F129L or G137R cyt $b$ mutations because these have never been observed in a 37-sample subset of isolates from the current study (data not shown) or reported in another study on V. inaequalis populations in the Michigan (20).

In this study, we found that nearly all orchard populations with reduced sensitivity to QoI fungicides had received two or more QoI fungicide applications per season (data not shown) for four or more years, which suggests that frequent QoI usage creates strong selective pressure for resistance. For example, orchard 117 (Table 1) had a population mean \%RG of 100.4 , with 20 (Table 1 ) of the 21 isolates having the $c y t b$ G143A mutation. In this extremely resistant orchard population, QoI fungicides had been applied three times per season for eight seasons according to pesticide application records. Similar levels of QoI resistance following intensive application schedules have been reported in other systems. It has been shown that more than 10 QoI applications in a kiwifruit planting had strongly selected for highly resistant isolates of $B$. cinerea populations (4), and six QoI applications annually in strawberry resulted in sufficient selective pressure for the development of resistant $B$. cinerea isolates (28). Intensive QoI usage for 9 years in Michigan was also sufficient for the development of $V$. inaequalis isolates with the $c y t b$ G143A mutation in several orchard populations (20). By comparison, there were 6 years of QoI use prior to the identification of $V$. inaequalis isolates with the $c y t b \mathrm{G} 143 \mathrm{~A}$ mutation in orchard populations in France (10).

In the present study, it was demonstrated that the $c y t b$ G143A mutation appears to be stable in isolates for six transfers in the absence of QoI selective pressure. Because a reversion or loss of mutation was never observed for any isolates during the course of the 6-month experiment, a reliable estimate of the duration allowing for a potential loss of the G143A mutation cannot fully be determined without further experimentation. Indeed, it has been reported that more than 30 similar clonal generations were needed before a loss of the G143A mutation or reversion to wild type were observed for the wheat pathogen Blumeria graminis f. sp. tritici (11). If the G143A mutation were to become absent over time in an individual or population, it might imply that there is a fitness cost associated with the mutation, and that mitochondria without the mutation would make the isolate more fit, so that individuals would have a selective advantage over resistant individuals. The results of other studies have shown little (21) or no (15) effects on fitness for qualitative QoI resistance in M. oryzae and Alternaria alternata, respectively. Hence, it is not surprising that the isolates in the present study maintained the mutation. Further analysis of $V$. inaequalis isolate fitness in regards to $c y t b$ genotype and monitoring of changes in the proportion isolates with qualitative resistance in an orchard setting where QoI applications have ceased would provide better insight into the stability of the G143A mutation.

Overall, the results of this study suggest that orchard populations of $V$. inaequalis with quantitative and qualitative resistance to QoI fungicides are present in the northeastern United States but that resistance is restricted or specific to the individual plantings. Although we found that many of the orchard populations examined in this study are not yet completely resistant, the potential exists for the development of practical resistance depending on continued QoI fungicide use, as suggested by Chapman et al. (6). Köller et al. (18) affirmed that QoI resistance management for $V$. inaequalis could be accomplished by limiting the number of QoI applications made seasonally. Such curtailment of QoI use, if practiced, could slow the selection of population members with both qualitative and quantitative resistance. However, further research is needed to understand the persistence of qualitative and even quantitative QoI 
resistance in the absence of QoI use over successive field seasons. From this point onward, apple producers in the northeastern United States should use QoI fungicides with caution until fungicide resistance testing is conducted on their local $V$. inaequalis orchard populations.

\section{Acknowledgments}

This work was supported, in part, by funds appropriated to New York State Agricultural Experiment Station, Cornell University. We thank W. Köller and D. Parker for providing isolates, for training the authors on many of the techniques utilized in the microbiological assays of the study, and for providing useful advice on the analysis and interpretation of data; our collaborators who assisted in the collection of field samples and fungicide use histories for evaluated orchards, including, J. Alicandro, P. Babcock, B. Baker, B. Bascombe, M. Belco, M. A. Bell, M. Biltonen, M. Borsick Herman, J. Bover, D. Breth, E. Brown, R. Bubier, J. Caroll, A. Clark, S. Clark, L. DeVoe, J. Dinardo, R. Drown, M. Ellis, M. Fargione, H. Faubert, H. Feller, M. Forrence, M. Forrence, M. Funk, Z. Goodband, D. Green III, A. Grobe, D. Hartley, L. Herring, A. Hill, C. Hodges, J. Ivison, C. Jaswell, E. Jensen, K. Karter, G. Koehler, E. Leadbeater, J. Lyman, A. Lynd, J. Matthews, J. Mitsiti, G. Morin, D. O'Connor, B. Olson, C. Petzoldt, D. Polseno, D. Rainer, D. Rosenberger, F. Santurri, R. Seem, M. Shannon, D. Simmons, B. Smith, R. Spitko, J. Steere, A. Sullivan, A. Teach, P. TenEyck, G. Thornton, D. Tower, F. Trail, B. Truncali, A. Tuttle, R. Wander, A. Wodehouse, S. Wood, M. Wright, K. Yoder, M. Zifchock, and S. Zonneville; and K. Abbot, W. Bacon, M. Ellis, J. Freier, N. Gottschall, C. Lehman, C. K. Poon, and M. Rosato for their assistance in the collection and preparation of and evaluation of isolates.

\section{Literature Cited}

1. Agnello, A. M., Landers, A. J., Rosenberger, D., Cox, K. D., Robinson, T. L., Carroll, J. E., Breth, D., Kain, D., Bellinder, R., Stiles, W., Cheng, L., Hoying, S., and Curtis, P. 2012. Pest management guidelines for commercial tree-fruit production 2012. Cornell Coop. Ext. N.Y. State Agric. Exp. Stn. Geneva.

2. Agrios, G. N. 2005. Plant Pathology, 5th ed. Elsevier Academic Press, Burlington, MA.

3. Anke, T., Oberwinkler, F., Steglich, W., and Schramm, G. 1977. The strobilurins-new antifungal antibiotics from the Basidiomycete Strobilurus tenacellus (Pers. Ex Fr.) Sing. J. Antibiot. 30:806-810.

4. Bardas G. A., Veloukas T., Koutita O., and Karaoglanidis G. S. 2010. Multiple resistance of Botrytis cinerea from kiwifruit to SDHIs, QoIs and fungicides of other chemical groups. Pest Manage. Sci. 66:967-973.

5. Bartlett, D. W., Clough, J. M., Godwin, J. R., Hall, A. A., Hamer, M., and Parr-Dobrzanski, B. 2002. The strobilurin fungicides. Pest Manage. Sci. 58:649-662.

6. Chapman, K. S., Sundin, G. W., and Beckerman, J. L. 2011. Identification of resistance to multiple fungicides in field populations of Venturia inaequalis. Plant Dis. 95:921-926.

7. Cox, K. D., Russo, N. L., Villani, S. M., Parker, D. M., and Köller, W. 2008. QoI qualitative resistance and CYP51A1 upstream anomalies in NY populations of the apple scab pathogen Venturia inaequalis. (Abstr.) Phytopathology. 98:S42.

8. Day, D. A., Whelan, J., Millar, A.H. Siedow, J. N., and Wiskich, J. T. 1995. Regulation of the alternative respiration pathway in plants and fungi. Aust. J. Plant Physiol. 22:497-509.

9. Färber, R. B. K., Chin, K. M., and Leadbitter, N. 2002. Sensitivity of Venturia inaequalis to trifloxystrobin. Pest Manage. Sci. 58:261-267.

10. Fontaine, S., Remuson, F., Fraissinet-Tachet, L., Micoud, A., Marmeisse, R., and Melayah, D. 2009. Monitoring of Venturia inaequalis harbouring the QoI resistance G143A mutation in French orchards as revealed by PCR assays. Pest Manage. Sci. 65:74-81.

11. Fraaije, B. A., Butters, J. A., Coelho, J. M., Jones, D. R., and Hollomon, D. W. 2002. Following the dynamics of strobilurin resistance in Blumeria graminis f. sp. tritici using quantitative allele-specific real-time PCR measurements with the fluorescent dye. Plant Pathol. 51:45-54.

12. Holb, I. J., Heijne, B., and Jeger, M. J. 2003. Summer epidemics of apple scab: the relationship between measurements and their implications for the development of predictive models and threshold levels under different dis- ease control regimes. J. Phytopathol. 151:335-343.

13. Jobin, T., and Carisse, O. 2007. Incidence of myclobutanil- and kresoximmethyl-insensitive isolates of Venturia inaequalis in Quebec orchards. Plant Dis. 91:1351-1358.

14. Jones, A. L., Aldwinkle, H. S. 1990. Compendium of Apple and Pear Diseases. American Phytopathological Society, St. Paul, MN.

15. Karaoglanidis, G. S., Luo, Y., and Michailides, T. J. 2011. Competitive ability and fitness of Alternaria alternata isolates resistant to QoI fungicides. Plant Dis. 95:178-182.

16. Kim, Y. S., Dixon, E. W., Vincelli, P., and Farman, M. L. 2003. Field resistance to strobilurin (QoI) fungicides in Pyricularia grisea caused by mutations in the mitochondrial cytochrome b gene. Phytopathology 93:891-900.

17. Köller, W. 1991. Fungicide resistance in plant pathogens. Pages 679-720 in: CRC Handbook of Pest Management in Agriculture, 2nd ed. CRC Press, Boca Raton, FL.

18. Köller, W., Parker, D. M., Turechek, W. W., Avila-Adame, C., and Cronshaw, K. 2004. A two-phase resistance response of Venturia inaequalis populations to the QoI fungicides kresoxim-methyl and trifloxystrobin. Plant Dis. 88:537-544.

19. Köller, W., Wilcox, W. F., Barnard, J., Jones, A. L., and Braun, P. G. 1997. Detection and quantification of resistance of Venturia inaequalis populations to sterol demethylation inhibitors. Phytopathology 87:184-190.

20. Lesniak, K. E., Proffer, T. J., Beckerman, J. L., and Sundin, G. W. 2011 Occurrence of QoI resistance and detection of the G143A mutation in Michigan populations of Venturia inaequalis. Plant Dis. 95:927-934.

21. Ma, B., and Uddin, W. 2009. Fitness and competitive ability of an azoxystrobin-resistant G143A mutant of Magnaporthe oryzae from perennial ryegrass. Plant Dis. 93:1044-1049.

22. Ma, Z., and Michailides, T. J. 2005. Advances in understanding molecular mechanisms of fungicide resistance and molecular detection of resistant genotypes in phytopathogenic fungi. Crop Prot. 24:853-863.

23. MacHardy, W. E. 1996. Apple Scab: Biology, Epidemiology, and Management. American Phytopathological Society, St. Paul, MN.

24. MacHardy, W. E., and Gadoury, D. M. 1989. A revision of Mills' s criteria for predicting apple scab infection periods. Phytopathology 79:304-310.

25. Mizutani, A., Miki, N., Yukioka, H., Tamura, H., and Masuko, M. 1996. A possible mechanism of control of rice blast disease by a novel alkoxyiminoacetamide fungicide, SSF126. Phytopathology 86:295-300.

26. Olaya, G., and Köller, W. 1999. Baseline sensitivities of Venturia inaequalis populations to the strobilurin fungicide kresoxim-methyl. Plant Dis. 83:274278.

27. Olaya, G., Zheng, D., and Köller, W. 1998. Differential Responses of Germinating Venturia inaequalis Conidia to Kresoxim-methyl. Pestic. Sci. 54:230-236.

28. Samuel, S., Papayiannis, L. C., Leroch, M., Veloukas, T., Hahn, M., and Karaoglanidis, G. S. 2011. Evaluation of the incidence of the G143A mutation and cytb intron presence in the cytochrome bc-1 gene conferring QoI resistance in Botrytis cinerea populations from several hosts. Pest Manage. Sci. 67:1029-1036.

29. Steinfeld, U., Sierotzki, H., Parisi, S., Poirey, S., and Gisi, U. 2001. Sensitivity of mitochondrial respiration to different inhibitors in Venturia inaequalis. Pest Manage. Sci. 57:787-796.

30. Tamura, H., Mizutani, A., Yukioka, H., Miki, N., Ohba, K., and Masuko, M 1999. Effect of the methoxyiminoacetamide fungicide,SSF129, on respiratory activity in Botrytis cinerea. Pestic. Sci. 55:681-686.

31. Vallières, C., Trouillard, M., Dujardin, G., and Meunier, B. 2011. Deleterious effect of the Qo inhibitor compound resistance-conferring mutation G143A in the intron-containing cytochrome $b$ gene and mechanisms for bypassing it. Appl. Environ. Microbiol. 77:2088-2093.

32. Van Den Bosch, F., Paveley, N., Shaw, M., Hobbelen, P., and Oliver, R. 2011. The dose rate debate: does the risk of fungicide resistance increase or decrease with dose? Plant Pathol. 60:597-606.

33. Zheng, D., Olaya, G., and Köller, W. 2000. Characterization of laboratory mutants of Venturia inaequalis resistant to the strobilurin-related fungicide kresoxim-methyl. Curr. Genet. 38:148-155.

34. Ziogas, B. N., Baldwin, B. C., Young, J. E. 1997. Alternative respiration: a biochemical mechanism of resistance to azoxystrobin (ICIA 5504) in Septoria tritici. Pestic. Sci. 50:28-34. 